Professor Dr.-Ing. Walter Frenzel 85 Jahre

Faserforseh. u. Textiltechnik 20 (1969) 9, S. 409.

DK $678.746 .22: 541.8: 532.13 .08: 535.36 .08$

\section{Dautzenberg, Herbert}

EinfluB der Lösungsmittelgüte auf das Verhalten linearer und verzweigter Makromoleküle in Lösung

Faserforsch. u. Textiltechnik 20 (1969) 9, S. '\&09-\$16. 8 Abb., 3 Tab., 23 I.it.

Zur Cntersuchung des Kinflusses der Lösungsmittelgüte auf das Verhalten von Makromolekũlen in Lösung wurden Lichtstreuungs- und Viskosităts messungen an linearen Polystyrolfraktionen und einem verzweigten Produkt durchgefóhrt. Die Variation der Lösungsmittelgūte erfolgte durch sukzessive Zugabe von Fällungsmittel. Die Krgebuisse werden anhand der bestehende Theorien uber das ausgeschlossene Volumen diskutiert.

DK $677.463 .021 .3: 677.463 .021 .319 .5: 532.13: 532.135$

Wulf, Karl, und Philipp, Burkihart

Rheologische Untersuchungen an Spinnlösungen

5. Mitt.: Zum Fließverhalten von Viskosespinnlösungen hoher Viskosität

Faserforsch. u. Textiltechnik 20 (1969) 9, s. 417-425. 6 Abb., 5 Tab., 29 lit.

Es wird über das Fließverhalten in langen und karzen Kapillaren bzw. Düsen von Viskosespinnlösungen berichtet, deren Anfangsviskositüten lurch inderung unterschicilicher chemischer Herstellungsparameter eingestellt wurdelt.

DK $677.463: 677.46 .021 .5: 546.2266^{\prime} 39: 539.216 .1: 537.533 .35$

Gensrich, Heinz Jïrgen, und Gröbe, Anneliese

Elektronenmikroskopische l'ntersuchungen an IJtradünnsehnitten von Celluloseregeneratfäden

Teil II. Die Struktur von Gelfäden, koaguliert in Ammoniumsulfatlösungen

Faserforsch. u. Textilfechnik 20 (1969) 9, $\$$.. $425-433$. 9 Abb., 55 l.it.

In Ammoniumsulfatlösungen ersponnene, darin regenerierte und zur $\mathrm{Be}$ wahrung des Gelzustandes indirekt durch Lösungsmittelaustausch bzw. Gefriertrocknung entwassserte Gelfaden werden untersucht und den ent sprechenden luftgetrockneten Proben gegenūbergestelit. Untersuchungsmethoden sind Uitradünnschnittechnik, Gefrierătztechnik, Oberflåchenabdrücke, Dichtemessung und $\mathrm{Hg}$-Porosimetrie. Die Morphologie der Gelfăden ist durch eine netzartige Anordnung von fibrillaren Strukturelementen gekennzeichnet, die einen Durchmesser von 90 bis $170 \hat{A}$ besitzen. Die Netzstruktur der Gelfāden schließt ein betrāchtliches Hohlraumvolumen ein, das beim direkten Trocknen der Gelfäden stark vermindert wird. Dennoch bleibt ein zusammenhãngendes Hohiraumsystem zurück. Die Errgebnisse über die Struktur der Gelfăden werden durch Untersuchungen übe Artefakte bei der Methacrylateinbettung gesichert.
DK 661.728.84:541.182:541.182.644

Pass, Ileinz, und Michels, Christoph

Beitrag zur Bestimmung des Gelteilchengehaltes in Viskosen Faserforseh. u. Textiltechnik 20 (1969) 9, s. 13/ı-137. 3 Abh., 2 Tab., 5 Lit.

Es wird von einer Kichmethode fûr das Gelteilchenzählgerait ZG 1 vom VEB T'R Dresden berichtet, die ohne Anwendung von Pollen oder Plastikkügelchen aufbauend auf den Gerätekonstanten gut reproduzierbare Partikelzāhlungen ermöglicht.

DK $661.728 .84: 677.463 .021 .5: 541.182: 658.562: 658.588 .002 .234$

Sendner, IIorst, Zimmermann, Karl, Büchner, Reinhard, und Gründling, Anneliese

Inhomogenitäten in Buchensulfitzellstoff-Viskosen und ihr Jinfluß auf den Spinn prozeß

Faserforsch. u. Textiltechnik 20 (1969) 9, s. $138-1 / 12$. 4 Abb., 2 Tab., 27 lit.

Die Arbeit befaßt sich mit dem Einfluß der Inhomogenitaiten auf den Viskosespinnproze日. Durch Partikelzăhlungen und Lichtdurchlässigkeits. messungen an Betriebsviskosen eines Viskosefaser-Großbetriebes wird der EinfluB des Zellstoff- Extraktgehaltes auf die Spinnsicherheit nachgewiesen. Des weiteren wird eine Methode zur Erarbeitung eines "Viskosepasses" zur trieblichen Viskosierprozesses dureh Partikelzăhluug lokalisiert werden können.

DK 677.494.674:677.494.675:677.494.061.382:677.494.061.3:535. . 27.2

Landgraf, Runhild, und Kaufmann, Siegfried

Untersuchungen mit einer Faserdreheinrichtung am Mikroskop

Teil 1: Apparatur und einige Anwendungen

Faserforsch. u. Textiltechnik 20 (1969) 9, s. $\quad 442-449$. 11 Abb., 17 Lit.

ks wird eine Faserdreheinrichtung beschrieben, die es gestattet, optische Messungen an beliebigen Querschnitten von Faserstoffen durchzuführen. Als Anwendung werden einige optische Konstanten von gequetsehter Poly amidselde und von s-yasern

DK $678.674^{\prime} 524^{\prime} 420: 543.257: 546.212: 658.562$

Dinse, IIans-Dieter, und Praeger, Konrad

Fine neue Betriebskontrollmethode \%ur Wasserbestimmung in Polyäthylenterephthalat

Faserforsch. u. Textiltechnik 20 (1969) 9, + . 49-450. 1 Abb., 1 Tab., 2 Lit.

DK $677.862 .25: 677.21: 547.458 .81: 541.124 .2: 546.714$

Lhlig, Egon, und Teichmann, Rainer

Über die Initiierung der Pfropfpolymerisation von Acrylnitril auf Cellulose durch Mangan (IV)

Faserforsch. u. Textiltechnik 20 (1969) 9, s. 451. 9 Lit.

DK 661.728.81:677.463.021.435:677.463.021.319

Treiber, Erich, und Lidbrandt, Ove

Zur Viskositätskorrigierung des Fillerwertes von Viskosen

Faserforsch. u. Textiltechnik 20 (1969) 9, S. 452-453. 2 Abb., 1 Tab., 5 Lit.

Neue Bücher S. $453-455$.

Patentschau S. $455-457$.

Literaturschau S. $457-460$. 

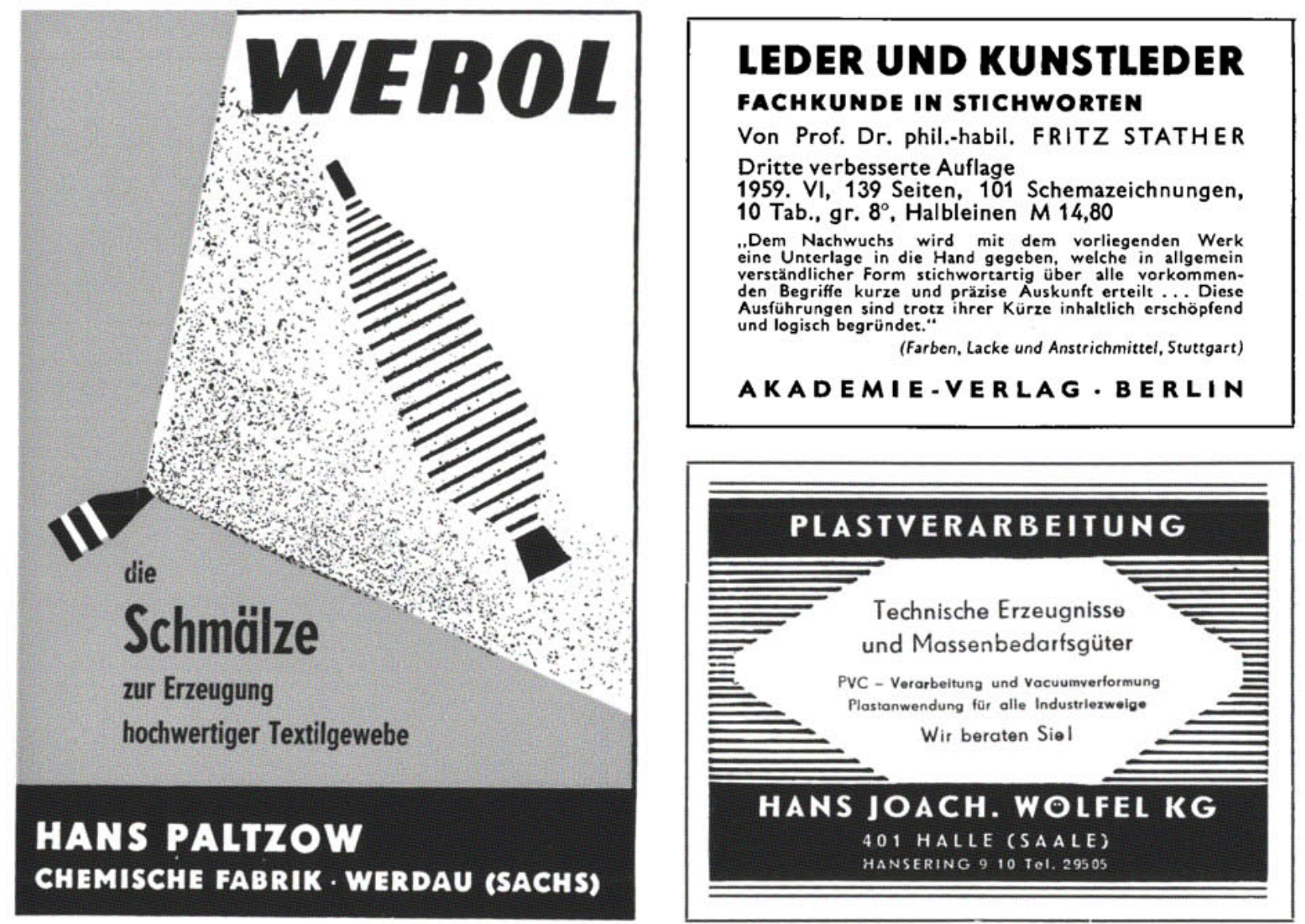

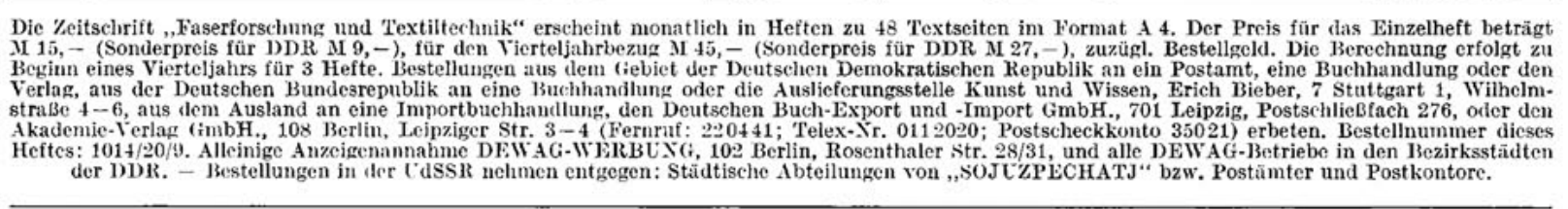

Herausgeber und verautwortlich für den Inhalt: Prof. Dr. Erich Correns, Institut für Faserstoff-Forschung der Deutschen Akademie der Wissenschaften zu Berlin, 153 Teltow-Sechof, Fernruf: Teltow 48 31; Prif. Dr.-Ing. habil. Wolfgang Bobeth, Institut für Technologie der Faseru der Deutschen Akademie der Wissenschaften zu Berlin, s01 Dresilen, Hohe Str. 6, Frrnruf: 44621 ; Prof. Dr.-Ing. Hans Böhringer, Institut für Textiltechuologie der Chemiefasern Rudol-

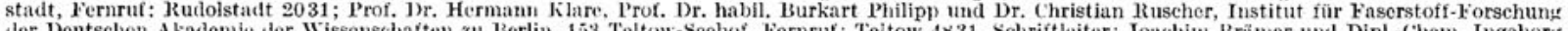

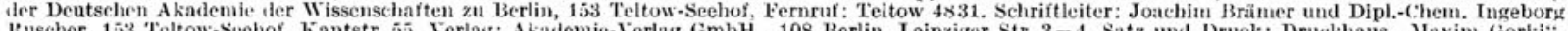

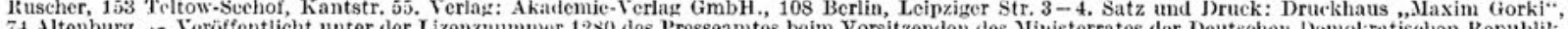

Manuskriptsendungen sind an einen der Herausgeber oder die Schriftleitung zu richten. Für Inhalt und Form gelten die , Kichtlinien für die Annahme und Abfassungen von Beiträgen", erhältlich von der Schriftleitung. Jie Verfasser grïBerer wissenschaftlieher Arbeiten erhalten aulser dem Honorar ein Ifeft und

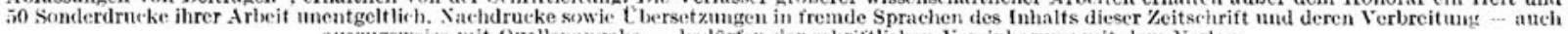
auszugsweise nit Quellenangabe - bedürfen der schriftlichen Vereinbarung mit dem Verlaz. 